Journal of Biological Systems, Vol. 3, No. 3 (1995) 779-787

(C) World Scientific Publishing Company

\title{
THRESHOLD QUANTITIES FOR INFECTIOUS DISEASES IN PERIODIC ENVIRONMENTS
}

\author{
J. A. P. HEESTERBEEK ${ }^{1, *}$ and M. G. ROBERTS ${ }^{2}$ \\ ${ }^{1}$ Agricultural Mathematics Group (GLW-DLO), P.O. Box 100, \\ 6700 AC Wageningen, The Netherlands \\ ${ }^{2}$ AgResearch, Wallaceville Animal Research Centre, P.O. Box 40063, \\ Upper Hutt, New Zealand
}

\begin{abstract}
In this short note we give threshold quantities that determine the stability of the infection-free steady state for periodic deterministic systems that describe the spread of infectious diseases in populations whose individuals can be divided into a finite number of distinct groups. We concentrate on "micro-parasitic" infections, but the theory was originally developed for helminth infections. As an example, we treat a simple model of a vector transmitted infection with periodic recruitment in the vector population.
\end{abstract}

Keywords: Deterministic epidemic model, periodic systems, threshold behaviour, basic reproduction ratio.

\section{Introduction}

The rigorous mathematical definition of $R_{0}$ for "micro-parasites" is based on the assumption that the infection develops as an autonomous process within the host [4]. One assumes that multiplication of the parasite within the host is so fast that additional re-infections play no part; the environment is considered to have no influence. One then regards generations of infected individuals and determines a positive operator that describes the next generation in terms of the present one and defines $R_{0}$ as the dominant eigenvalue of this (next-generation) operator. The fact that we may assume autonomy within the host once infection has occured, justifies the use of an age-representation for the development of the infection within the host and one can meaningfully discuss the infectiousness of the host individual as a function of the time elapsed since infection took place. To keep track of the infectious "output" of the host, one need only to know the age-of-infection. $R_{0}$ has the interpretation of expected number of newly infected hosts caused by a typical infected host during its entire period of infectivity in a population consisting of susceptibles only.

\footnotetext{
*Author for correspondence, e-mail: heesterbeek@glw.agro.nl
} 
In [4], the susceptible population into which the parasite is introduced is considered to be in a demographic steady state at the moment of invasion. The steady state in absence of infection is an explicit part of the definition of the next-generation operator. The theory developed in [4] uses the fact that in a constant environment, the operator is time-independent. In many cases however, the environment can have a non-negligible influence on the density of the susceptible population, and it is often unrealistic for the susceptible population to be considered constant even in the absence of infection. For example, in many host-vector systems, recruitment into the vector population (emergence of adults) is temperature dependent, and the vector population size or density will show seasonal oscillations [1].

The theory discussed in this paper was developed as an attempt to give threshold quantities for the invasion of helminth parasites. In that case, the environment not only influences the susceptible population, but, more importantly, also the infectious "output" of an infected host. Macroparasites have a distinct life-cycle with different stages in different environments, and the rise in the level of the infection inside a host is in general exclusively due to re-infection. Often, the environmental influences on survival and reproduction of the various stages in the life-cycle are, in approximation, periodic. Therefore, it is, for helminth infections, important to have threshold quantities that explicitly take periodic survival and reproduction for each life-stage into account. This theory was developed in [9], and applied to the evaluation of control programmes for nematode infections of ruminants in [11].

In Sec. 2 of the present paper, this theory is applied to define threshold quantities that determine the ability of a microparasite to invade a susceptible population that is (i) not in a constant but in a periodic demographic state prior to invasion, and (ii) where other possible periodicities can be taken into account, e.g. in the rates of removal from the infective stage. In Sec. 3, we discuss in detail a simple model of vector transmitted microparasitic infections, where the emergence of adults is described by a periodic function.

The related problem of characterising the Malthusian parameter in branching processes where the reproduction of individuals is allowed to vary periodically with time, was first studied by Jagers and Nerman for populations consisting of a single type in [10]. We do not go into the differences between the two approaches here.

\section{Three Threshold Quantities}

\subsection{Autonomous Case}

To better understand the periodic case, we start by very briefly describing the ingredients to calculate $R_{0}$ in a constant environment, when the transmission-dynamics in a population consisting of $n$ different groups is described by a simple compartmental system. Let $x=\left(x_{1}, \ldots, x_{n}\right)^{T}$ be the vector of population densities of those infected in the various groups, let the matrix $M(S)$ contain the transmission terms $m_{i j}(S)$ that describe the average number of new infections per unit of time arising 
among susceptibles of group $i$, caused by an infected individual of group $j$. This matrix depends on the density $S=\left(S_{1}, \ldots, S_{n}\right)$ of the susceptible population, which is assumed to be in a constant demographic steady state prior to the introduction of the parasite. Finally, let $D$ be the diagonal matrix that describes the removal rates out of the infected class for the members of the $n$ groups. We regard the following system

$$
\frac{d x}{d t}(t)=M(S) x(t)-D x(t)=: A x(t), \quad x(0)=x_{0}
$$

where $S^{*}$ is the unique steady state solution of

$$
\frac{d S}{d t}=g(S)
$$

that describes the dynamics of the susceptible subpopulations in the absence of infection. The next-generation operator for this particular situation is given by the matrix

$$
K\left(S^{*}\right)=M\left(S^{*}\right) D^{-1} .
$$

One now defines $R_{0}=r(K)$, the spectral radius of $K\left(S^{*}\right)$, or dominant eigenvalue of $K\left(S^{*}\right)$ since $K$ is positive. It is easy to show, see e.g. [8], that

$$
r(K)<1 \Leftrightarrow s(A)<0
$$

where $s(A)$ is the spectral bound of $A$, i.e. the eigenvalue with the largest real part. Therefore, $r(K)<1$ implies local asymptotic stability of the trivial, i.e. infectionfree, steady state of $(2.1)$, and $r(K)>1$ implies that the infection-free steady state is unstable and invasion can be successful.

Of course, any integer power of $r(K)$ has the same property, and for infections with a definite life-cycle consisting of $k$ stages one could argue that $r(K)^{k}$ would be a better definition for $R_{0}[7,9]$, but see [5] for caveats. With a definite lifecycle we mean that it is a biological necessity that multiple host-types are involved and that intra-type transmission is in general not possible. Helminths thus have a clear life-cycle, but vector transmitted microparasites also qualify, where sexual reproduction usually takes place in the vector only and where vectors cannot infect vectors directly, and mutatis mutandis for the host species. For sexually transmitted infections in heterosexual populations, where one could be tempted to separate infections of males and females, and subsequently regard the "cycle" male infects female infects male etc., it is not a biological necessity for the parasite to have these two host-types, but merely a modelling assumption. These infections are therefore examples where one would not speak of a definite life-cycle for the parasite. For microparasites, we will adhere to the cycle idea only in our host-vector example.

\subsection{Periodic Case 1 (Floquet Multiplier)}

We now turn to the periodic case. Let the matrices $M(S)$ and $D$ in (2.1) be timedependent and periodic with the same period. For convenience, we scale the period 
to 1 . Then,

$$
\frac{d x}{d t}(t)=M(S)(t) x(t)-D(t) x(t)=: A(t) x(t), \quad x(0)=x_{0}
$$

with $M(S)(t+1)=M(S)(t), D(t+1)=D(t)$ for all $t \geq 0$, and consequently $A(t+1)=A(t)$, and where $S(t)$ is the periodic solution of

$$
\frac{d S}{d t}(t)=g(t, S(t))
$$

with function $g(\cdot, S)$ periodic with period 1 .

We will discuss three different threshold quantities, two generally applicable ones and one special quantity. For the proofs we refer the reader to [9]. First consider the standard mathematical way of dealing with the stability of the trivial steady state of (2.4). Let $\Phi(t)$ be the standard fundamental solution of (2.4), i.e.

$$
\frac{d \Phi}{d t}(t)=A(t) \Phi(t), \quad \Phi(0)=I
$$

In our situation $\Phi$ is a positive matrix, i.e. all entries of $\Phi$ are $\geq 0$. The solution to $(2.4)$ can be written as $x(t)=\Phi(t) x_{0}$. We can write

$$
\Phi(t+1)=\Phi(t) E
$$

where $E$ is a constant (i.e. time-independent) $n * n$ matrix, whose eigenvalues are uniquely determined by $A(t)$, see $[3,6]$ for standard results in this direction. Setting $t=0$, we find $E=\Phi(1)$, and moreover that in our case $E$ is a positive matrix. The dominant eigenvalue of $E$ (or dominant Floquet multiplier) determines the stability of the trivial steady state. From a mathematical point of view therefore, a sensible threshold quantity for periodic systems like (2.4) is the dominant eigenvalue $\lambda(E)$ of $E$.

In the autonomous case (2.1), the fundamental solution is given by $\Phi(t)=$ $\exp (t A)$, so $E=\exp (A)$ and one can then easily see that $r(K)<1 \Leftrightarrow \lambda(E)<1$.

\subsection{Periodic Case 2 (Basic Reproduction Ratio)}

Although $\lambda(E)$ is probably the "easiest" of the three threshold quantities to calculate numerically, its implicit definition makes it not a suitable candidate for the development of meaningful approximating expressions. We therefore introduce a second quantity related to $r(K)$ in the autonomous case.

First define $f_{i}(t)>0, i \in\{1, \ldots, n\}$, as the average duration of the infective stage for an infected individual of group $i$, given that the individual became infected at time $t$. If we write $F(t)=\operatorname{diag}\left(f_{1}(t), \ldots, f_{n}(t)\right)$, for the diagonal matrix of average durations of the infective period, then $F=D^{-1}$ in the case that $D$ does not depend on time. For the case that $D$ depends on time

$$
f_{i}(t)=\int_{0}^{\infty} e^{-\int_{0}^{a} d_{i}(t+z) d z} d a
$$


and one can easily show that $F(t)$ obeys

$$
\frac{d F}{d t}(t)=D(t) F(t)-I, \quad F(0)=F(1)
$$

Let $F(0)=F(1)=F_{0}$, and define a matrix $\kappa$ as

$$
\kappa:=C^{-1} \int_{0}^{1} F(t) M(S)(t) \Phi(t) d t
$$

where

$$
C=\int_{0}^{1} \Phi(t) d t .
$$

The rationale of arriving at the definition of $\kappa$ is given in [9]. What is important for our purpose is that (i) in the autonomous case $\kappa$ is similar to $K, \kappa \sim K$, where $K$ is given by (2.3), and therefore in particular $\kappa$ and $K$ have the same spectral radius, and (ii) one can prove that in the nonautonomous case, the spectral radius of $\mathrm{k}$ has the same threshold behaviour as $\lambda(E)$ :

$$
\lambda(E)<1 \Leftrightarrow r(\kappa)<1 .
$$

The dominant eigenvalue $r(\kappa)$ of $\kappa$ is a threshold quantity for the stability of the trivial steady state of (2.4), and therefore a suitable candidate for $R_{0}$ in periodic environments. The biological interpretation of $R_{0}=r(\kappa)$ then coincides with the usual one. Save for a normalisation, $\kappa$ is, in analogy to models in demography, the integral over the reproduction function $F M(S)$, i.e. the product of fertility $M(S)$ with life expectancy $F$. The approximation possibilities of (2.8) relative to those of the more implicit definition of $E$, seem to be an improvement.

\subsection{Periodic Case for Parasites with Clear Life-Cycle}

In the special case that the parasite has a clear life-cycle, with different stages taking place in different environments as a biological necessity, we can define a third related quantity $P$. Regard a parasite whose life-cycle consists of $k$ consecutive stages, where the host of stage $i$ can only infect the host of stage $i+1$, and hosts of stage $k$ infect hosts of stage 1 . If we regard this as a special kind of heterogeneous population with $k$ types of individuals (usually different species) then we can use the descriptions (2.1) and (2.4) above. For convenience regard the case $k=2$, for an example of a vector transmitted parasite. Then the matrix $M(S)$ has the special form

$$
M(S)=\left(\begin{array}{cc}
0 & m_{12}(S) \\
m_{21}(S) & 0
\end{array}\right) .
$$

With $k$ types there will only be non-zero elements $m_{i j}$ on the sub-diagonal, and in position $1 k$ of $M(S)$. The following notation for these elements is then unambiguous: 
$m_{i j}(S)=: m_{j}(S)$. In the autonomous case, direct calculation of $R_{0}=r(K)^{2}$ leads to

$$
R_{0}=\prod_{i=1}^{k} \frac{m_{i}(S)}{d_{i}}
$$

In the periodic case we define a quantity $P$ as a direct generalisation of (2.9)

$$
P:=\prod_{i=1}^{k} \frac{\int_{0}^{1} f_{i+1}(t) m_{i}(S)(t) x_{i}(t) d t}{\int_{0}^{1} x_{i}(t) d t}
$$

where $x(t)$ is the solution of (2.4) with the eigenvector corresponding to $\lambda(E)$ (technical condition, see [9]) as the initial condition. Then $P$ has the same threshold behaviour as $\lambda(E)$ and $R_{0}$ because one can prove

$$
\lambda(E)<1 \Leftrightarrow P<1 .
$$

From a computational point of view, $P$ is not an immediate option, since evaluating it requires calculating $\lambda(E)$ as a first step. However, $P$ is interesting because it has a different biological interpretation to $R_{0}$, and its explicitness might be exploited in approximations. $P$ can be interpreted as the expected number of years of host-infectivity that is produced per year of host infectivity. In the autonomous case, $P=R_{0}$, the interpretations coincide because the average lengths of the infective periods for the various stages are constant. In the non-autonomous case however, the duration of the infective period depends on the time $t$ that the individual became infected, and then the interpretations of $P$ and $R_{0}$ are not the same, and $P$ and $R_{0}$ can have different values. and $m$

One can, as a first simple attempt, approximate $P$ by Taylor expansion of $f$

$$
P \approx \prod_{i=1}^{k}\left(f_{i+1}(0) m_{i}(0)+\left[\frac{d f_{i+1}}{d t}(0) m_{i}(0)+f_{i+1}(0) \frac{d m_{i}}{d t}(0)\right] \frac{\int_{0}^{1} t x_{i}(t) d t}{\int_{0}^{1} x_{i}(t) d t}\right) .
$$

If one could estimate the values of $f$ and $m$ (and their derivatives) in the beginning of the period, then the only unknown in (2.11) is the quotient of integrals in the last term. This quotient however, can be interpreted as the approximate time in the period that the epidemic reaches its maximum prevalence for the various parasite stages and this information might be obtainable from data. For example, in the host-vector case, the terms would be approximately the time that the epidemic peaks in the host population and the vector population respectively. This type of data is mentioned in, for example, $[1$, Sec. 14.4] with respect to malaria, see also Sec. 3 below.

\section{Host-Vector Example}

We consider a model for a vector transmitted infection, akin to the Ross malaria model and a rudimentary version of a model for African Horse Sickness (caused by 
a virus transmitted by midges). Much more detailed versions of a different model of this host-vector system involving periodicity are currently being studied with emphasis on the computation of $R_{0}$ [12].

We assume changes in the host population occur at a much slower time-scale than changes in the vector population and consider the total host population as constant. Since we want to characterise invasion, we assume from the start that all hosts and vectors are susceptible prior to introduction of the infection. Let $x_{1}(t)$ denote the fraction of the host population that is infected at time $t$, and let the density of the susceptible and infected vector population be described by $s(t)$ and $x_{2}(t)$, respectively. Let $r$ be the removal rate of hosts, $\mu$ the death-rate of vectors, $b$ the transmission-rate constant for infection of hosts by vectors, and $\beta$ the transmission-rate constant for the infection of vectors by hosts. Finally, let $\rho(t)$ be the recruitment rate of the vector at time $t$. Note that we can allow all parameters mentioned to be periodic functions. We limit ourselves to the recruitment rate. Let $x=\left(x_{1}, x_{2}\right)^{T}$ be the vector of infectives.

The above leads to the following specification of system (2.4)-(2.5).

$$
\frac{d x}{d t}(t)=\left(\begin{array}{cc}
-r & \frac{b}{s(t)} \\
\beta s(t) & -\mu
\end{array}\right) x(t)
$$

with the dynamics of the susceptible vector population $s(t)$ in the absence of infection described by

$$
\frac{d s}{d t}=\rho(t) s(t)-\mu s(t) .
$$

In the autonomous situation we find from Sec. 2, or directly from the interpretation, that

$$
R_{0}=\frac{b \beta}{\mu r}
$$

Consider the case that

$$
\rho(t)=\mu(1+\delta \cos (2 \pi t))
$$

The solution of (3.2) is then

$$
s(t)=s(0) e^{\mu \delta \sin (2 \pi t)}
$$

We write $\varepsilon=\mu \delta$ and note that $s(t)=s(0)+s(0) \varepsilon \sin (2 \pi t)+o(\varepsilon)$, and assume realistically that $\varepsilon<1$ (see for example simulations in [1]).

Two of the three threshold quantities formulated in Sec. 2, have to be evaluated numerically, since there is no general procedure to calculate fundamental matrix solutions of (2.4) directly. The procedure to numerically compute the standard fundamental matrix solution of (3.1) is to solve (3.1) twice over one period, with the two standard unit vectors of $\mathbb{R}^{2}$ as consecutive initial conditions and taking the 
resulting vectors as columns of $\Phi(t)$. Then $E=\Phi(1)$, and the threshold quantity is $\lambda(E)$. The second quantity is defined as $R_{0}=r(\kappa)^{2}$ where

$$
\kappa=C^{-1} D^{-1} \int_{0}^{1} M(S)(t) \Phi(t) d t
$$

with $D=\operatorname{diag}(r, \mu)$ and

$$
M(S)(t)=\left(\begin{array}{cc}
0 & \frac{b}{s(t)} \\
\beta s(t) & 0
\end{array}\right)
$$

and $s(t)$ given by (3.4). Also $r(\kappa)$ has to be evaluated numerically.

Finally, we turn to $P$. Here we can get an idea of the influence of $\varepsilon$ on the threshold, if $\varepsilon$ is small. The approximation (2.11) of $P$ for $\operatorname{small} \varepsilon$, leads to the following expression, where we write $\grave{\varepsilon}=2 \pi \varepsilon$,

$$
P=\frac{b \beta}{\mu r}+\frac{\grave{\varepsilon} b \beta}{\mu r}\left(\frac{\int_{0}^{1} t x_{1}(t) d t}{\int_{0}^{1} x_{1}(t) d t}-\frac{\int_{0}^{1} t x_{2}(t) d t}{\int_{0}^{1} x_{2}(t) d t}\right)+o(\grave{\varepsilon})
$$

or

$$
P=R_{0}+\grave{\varepsilon} R_{0}\left(T_{h}-T_{v}\right)+o(\grave{\varepsilon})
$$

where $R_{0}$ is given by (3.3). The constants $T_{h}$ and $T_{v}$ can be interpreted as the time within the period that the infection reaches maximum prevalence in the host and vector population, respectively. If these constants can be estimated, or at least their difference, and if $\varepsilon$ can be estimated from observations of the vector population, then this estimate for $P$ can give an indication about the influence on $R_{0}$ of periodicity in the vector population.

Anderson and May [1] discuss data on $T_{h}$ and $T_{v}$ for malaria. They cite two papers from [2] showing that the "maximum prevalence among mosquitoes follows both the peak in prevalence within humans and the peak in mosquito abundance". While this may of itself be understandable from a biological point of view (K. Dietz, personal communication), it nevertheless suggests that, for malaria, $T_{h}-T_{v}<0$, and we therefore conclude that for small $\varepsilon$, the periodicity can lead to a lower value of the threshold quantity than in the autonomous case.

\section{Discussion}

We have shown that, in periodic environments, there are at least three ways of defining a threshold quantity for the invasion of parasites, be they micro or macroparasites. The dominant eigenvalue of matrix $E$, associated with the fundamental matrix solutions to the linearised system describing the transmission dynamics, seems to be the best option from a numerical point of view. The ad hoc definition of $P$ seems to give the best possibilities for approximation. It might turn out that $P$ is even a better option than $R_{0}=r(\kappa)$ numerically, because, even though 
the computation of $P$ has the computation of $\lambda(E)$ as a first step, the remaining numerical procedure is straightforward. In case of $R_{0}=r(\kappa)$ however, matrices have to be inverted that may turn out to be close to singular, causing numerical instabilities.

Instead of the obvious goal to generalise the "theory" to heterogeneity involving arbitrary state space, or to develop similar results for chaotic environments (for example based on dominant Lyapunov exponents), a more immediate aim should be to develop meaningful approximations to the quantities defined in this paper. The simple approximation of $P$ given above, that leads to expressions involving unknown functions that in combination with others nevertheless allow a biological interpretation and estimation, is an example of the possibilities. It also has to be investigated whether assumptions can be made that could lead to analytical calculation of the quantities defined, in analogy to for example the assumption of separable mixing, that in the case of $R_{0}$ for general state-spaces, leads to an explicit expression of $R_{0}$ [4].

\section{References}

[1] Anderson R. M. and May R. M., Infectious Diseases of Humans: Dynamics and Control (Oxford Univ. Press, 1991).

[2] Boyd M. F. (ed.), Malariology (Saunders, Philadelphia, 1949).

[3] Coddington E. A. and Levinson N., Theory of Ordinary Differential Equations (McGraw-Hill, New York, 1955).

[4] Diekmann O., Heesterbeek J. A. P. and Metz J. A. J., On the definition and the computation of the basic reproduction ratio $R_{0}$ in models for infectious diseases in heterogeneous populations, J. Math. Biol. 28 (1990) pp. 365-382.

[5] Diekmann O., Heesterbeek J. A. P. and Metz J. A. J., The legacy of Kermack and McKendrick. In Epidemic Models, their Structure and Relation to Data, ed. by D. Mollison (Cambridge Univ. Press, 1995) pp. 95-115.

[6] Grimshaw R., Nonlinear Ordinary Differential Equations (Blackwell Scientific Publications, Oxford, 1990).

[7] Hasibeder G., Heterogeneous disease transmission: estimating the basic reproduction number from prevalences. In Infectious Human Diseases, ed. by V. Isham and G. Medley (Cambridge Univ. Press, 1995) in press.

[8] Heesterbeek J. A. P., $R_{0}$, PhD thesis, University of Leiden, 1992.

[9] Heesterbeek J. A. P. and Roberts M. G., Threshold quantities for helminth infections, J. Math. Biol. 33 (1995) pp. 415-434.

[10] Jagers P. and Nerman O., Branching processes in periodically varying environment, Ann. Prob. 13 (1985) pp. 254-268.

[11] Roberts M. G. and Heesterbeek J. A. P., The dynamics of nematode infections of farmed ruminants, Parasitology 110 (1995) pp. 493-502.

[12] Lord C. C., Woolhouse M. E. J., Heesterbeek J. A. P. and Mellor P. S., Vector-borne diseases and the basic reproduction number: a case study of African horse sickness, Med. Vet. Entom., in press. 